\title{
Intron-Based Single Transcript Unit CRISPR Systems for Plant Genome Editing
}

\author{
Zhaohui Zhong ${ }^{1 \dagger}$, Shishi Liu ${ }^{1 \dagger}$, Xiaopei Liu', Binglin Liu', Xu Tang ${ }^{1}$, Qiurong Ren ${ }^{1}$, Jianping Zhou', Xuelian Zheng ${ }^{1}$,
} Yiping $\mathrm{Qi}^{3,4^{*}}$ and Yong Zhang ${ }^{1,2^{*}}$ (i)

\begin{abstract}
Background: Expression of either Cas9 or Cas12a and guide RNAs by a single Polymerase II (Pol II) promoter represents a compact CRISPR expression system and has many advantages for different applications. In order to make this system routine in plant biology, engineering efforts are needed for developing and optimizing such single transcript unit (STU) systems for plant genome editing.
\end{abstract}

Results: To develop novel intron-based STU (iSTU) CRISPR system (STU CRISPR 3.0), we first evaluated three introns from three plant species for carrying guide RNAs by using an enhanced green fluorescence protein (eGFP) system in rice. After validation of proper intron slicing, we inserted these gRNA-containing introns into the open reading frames (ORFs) of Cas9 and Cas12a for testing their genome editing capability. Different guide RNA processing strategies have been tested for Cas9 and Cas12a. We demonstrated singular genome editing and multiplexed genome editing with these iSTU-Cas9 and iSTU-Cas12a systems.

Conclusion: We developed multiple iSTU-CRISPR/Cas9 and Cas12a systems for plant genome editing. Our results shed light on potential directions for further improvement of the iSTU systems.

Keywords: STU CRISPR 3.0, Cas9, Cas12a, iSTU, Rice

\section{Background}

Sequence-specific nucleases (SSNs), such as zinc finger nucleases (ZFNs), TAL effector nucleases (TALENs) and clustered regularly interspaced short palindromic repeats (CRISPR), have promoted plant reverse genetics and breeding in the past decade (Puchta. 2017, Voytas. 2013). DNA targeting by ZFNs and TALENs is protein-based, which requires co-expression of two protein monomers either separately by two Polymerase II (Pol II) promoters or by one Pol II promoter with a ribosome skipping peptide generating two proteins from a single mRNA (Qi et al. 2013, Shan et al. 2013a, Zhang et al. 2013). Thanks to simple RNA-based DNA targeting, CRISPR/Cas9 and Cas12a systems become leading systems for plant genome

\footnotetext{
*Correspondence: yiping@umd.edu; zhangyong916@uestc.edu.cn ${ }^{\dagger}$ Zhaohui Zhong and Shishi Liu are authors contributed equally to this work. ${ }^{3}$ Department of Plant Science and Landscape Architecture, University of Maryland, College Park, MD 20742, USA

'Department of Biotechnology, School of Life Sciences and Technology, Center for Informational Biology, University of Electronic Science and Technology of China, Room 216, Main Building, No. 4, Section 2, North Jianshe Road, Chengdu 610054, People's Republic of China Full list of author information is available at the end of the article
}

editing (Zhang et al. 2019). Application of CRISPR technologies in plants often requires the co-expression of the Cas protein and guide RNAs (gRNAs). There are generally four expression strategies for CRISPR/Cas9 or Cas12a (Zhang et al. 2019). The first strategy relies on a mixed dual promoter system in which the Cas gene is expressed by a Pol II promoter and gRNA is expressed by a Pol III promoter like U6 or U3. This system was used when CRISPR/Cas9 was first demonstrated in plants (Jiang et al. 2013, Li et al. 2013, Nekrasov et al. 2013, Shan et al. 2013b, Zhong et al. 2019). The second strategy relies on a dual Pol II promoter system where the Cas gene and the gRNA are separately expressed by Pol II promoters (Cermak et al. 2017, Tang et al. 2017, Zhong et al. 2018). The third strategy is based on a single Pol II bidirectional promoter which expresses the Cas9 gene and the gRNA in opposite directions, and this system was demonstrated in plants very recently (Ren et al. 2019). The fourth strategy expresses the Cas gene and the gRNA in a single transcript unit (STU). The STU system is the most compact expression system, rendering both CRISPR components under coordinated expression by a single Pol II promoter 
and a single terminator (Tang et al. 2019, Tang et al. 2016). The STU system is very appealing for applications that require inducible or tissue specific expression, as well as for CRISPR based transcriptional regulations in plants (Lowder et al. 2015).

Precise processing of gRNAs is key to the success of a STU CRISPR system. Previously, processing of a single guide RNA (sgRNA) for Cas9 has been demonstrated with a hammer head (HH)-hepatitis delta virus (HDV) dual ribozyme system (Gao \& Zhao. 2014), a tRNA system (Xie et al. 2015), or Csy4 which is a sequence-specific RNase (Tsai et al. 2014). Building on these previous studies, the first demonstration of a CRISPR/Cas9 STU system in plants employed $\mathrm{HH}$ ribozyme for precise processing of single guide RNAs (sgRNAs) (Tang et al. 2016). More recently, newer versions of STU systems were developed for plant genome editing where many sgRNA processing strategies have been tested, including Csy4 (Tang et al. 2019) and tRNA (Tang et al. 2019, Wang et al. 2018). Strikingly, expressing sgRNAs without any additional processing also worked in rice (Mikami et al. 2017, Wang et al. 2018). In the case of the STU-Cas12a system, CRISPR RNA (crRNA) processing is either based on Cas12a's self-processing of direct repeat (DR) CRISPR array (Tang et al. 2019) or HH-HDV ribozyme-based processing (Wang et al. 2018). In these STU systems, the gRNA cassettes were positioned at the $3^{\prime}$ end of the Cas9 gene, separated by a Poly (A) sequence or a linker sequence. In both cases, the Cas gene is not immediately terminated by a conventional terminator, which allows for transcription of the gRNA component. Such a design however may have negative effects on Cas mRNA maturation and gRNA stability. Therefore, it is worthwhile pursuing additional STU strategies, such as positioning gRNAs (e.g. sgRNAs for Cas9 or crRNAs for Cas12a) at the $5^{\prime}$ end of the Cas gene or within a Cas open reading frame (ORF). For these strategies to work, it is inevitable to rely on introns for the expression gRNAs, whether gRNAs for Cas9 or crRNA for Cas12a. Only one recent study has explored the use of introns for gRNA expression in plants (Ding et al. 2018). In that study, a gRNA-containing intron was positioned at the 5' UTR of the Cas gene and the authors successfully demonstrated genome editing in rice with Cas9 and Cas12a (Ding et al. 2018). However, it could be tricky to apply this system into other plants as the modified 5' UTR intron may not be compatible with the promoter of choice. Since $5^{\prime}$ UTR introns can often affect transcription and translation (Akua \& Shaul. 2013, Cenik et al. 2010, Gallegos \& Rose. 2017), it adds another layer of complexity when modifying them because the compatibility of the modified $5^{\prime}$ UTR intron with the promoter of choice has to be empirically tested in a case by case scenario.
The majority of plant endogenous genes contain introns that break apart their ORFs. These introns are embedded in gene bodies and provide numerous opportunities to be repurposed to carry gRNA cassettes. Using introns embedded in gene bodies for gRNA expression would allow us to develop reliable STU CRISPR systems that are independent of promoters of choice, thus making the system highly modular and can be readily used with all kinds of promoters in different plant species. Here, we inserted sgRNA or crRNA into such introns and showed these modified introns did not affect splicing, resulting fully functional proteins. We then inserted such gRNA-carrying introns into the ORFs of Cas9 and Cas12a for testing plant genome editing. We compared three introns from three different plant species and multiple gRNA processing mechanisms. Our work not only demonstrated versatile intron-based STU (iSTU) CRISPR systems (STU CRISPR 3.0) for CRISPR/Cas9 and Cas12a applications in rice, a major crop, but also shed light on future improvement and applications of this technology.

\section{Results \\ Evaluation of Three Introns with an eGFP Reporter System in Rice Protoplasts}

To develop iSTU systems, we inserted three introns into the OFR of an enhanced green fluorescence protein (eGFP) gene which was drive by the maize ubiquitin 1 (pZmUbi1) promoter (Fig. 1a). The three introns tested were a 189-bp StIV2 intron (inS) from potato, a 290-bp OsCDPK2_1 intron (inO) from rice and a 190-bp RcCAT_ 1 intron (inR) from castor bean. We modified the three introns by inserting tRNA-sgRNA-tRNA cassette (tRNA), HH ribozyme-sgRNA-HH ribozyme (RZ) or null processing sgRNA cassette (NU) in between the $5^{\prime}$ splice site and the branch site (Fig. 1a). A total of nine configurations (three introns by three sgRNA processing mechanisms) were tested. In addition, an intron-less eGFP was included as a positive control and an eGFP with a frame shift mutation $(\triangle \mathrm{eGFP})$ was included as a negative control. We transfected rice protoplasts with these $11 \mathrm{~T}$-DNA expression vectors and imaged transfected cells under a fluorescence microscope 2 days after transfection. As expected, majority of protoplasts transfected with the eGFP positive control vector showed GFP signal, while the protoplasts transfected with the $\triangle \mathrm{eGFP}$ negative control had no GFP signal (Fig. 1b). All three sgRNA processing systems, when positioned in the OsCDPK2_1 intron, did not affect intron splicing as most of transfected cells showed GFP signals (Fig. 1b). The same was true for the other two modified introns with three sgRNA processing systems as all six vectors resulted in GFP signal in transfected protoplasts (Additional file 1: Figure S1). We quantified relative fluorescence intensity and found the three modified intron systems (inS, inO and inR) all had equivalent levels of GFP 


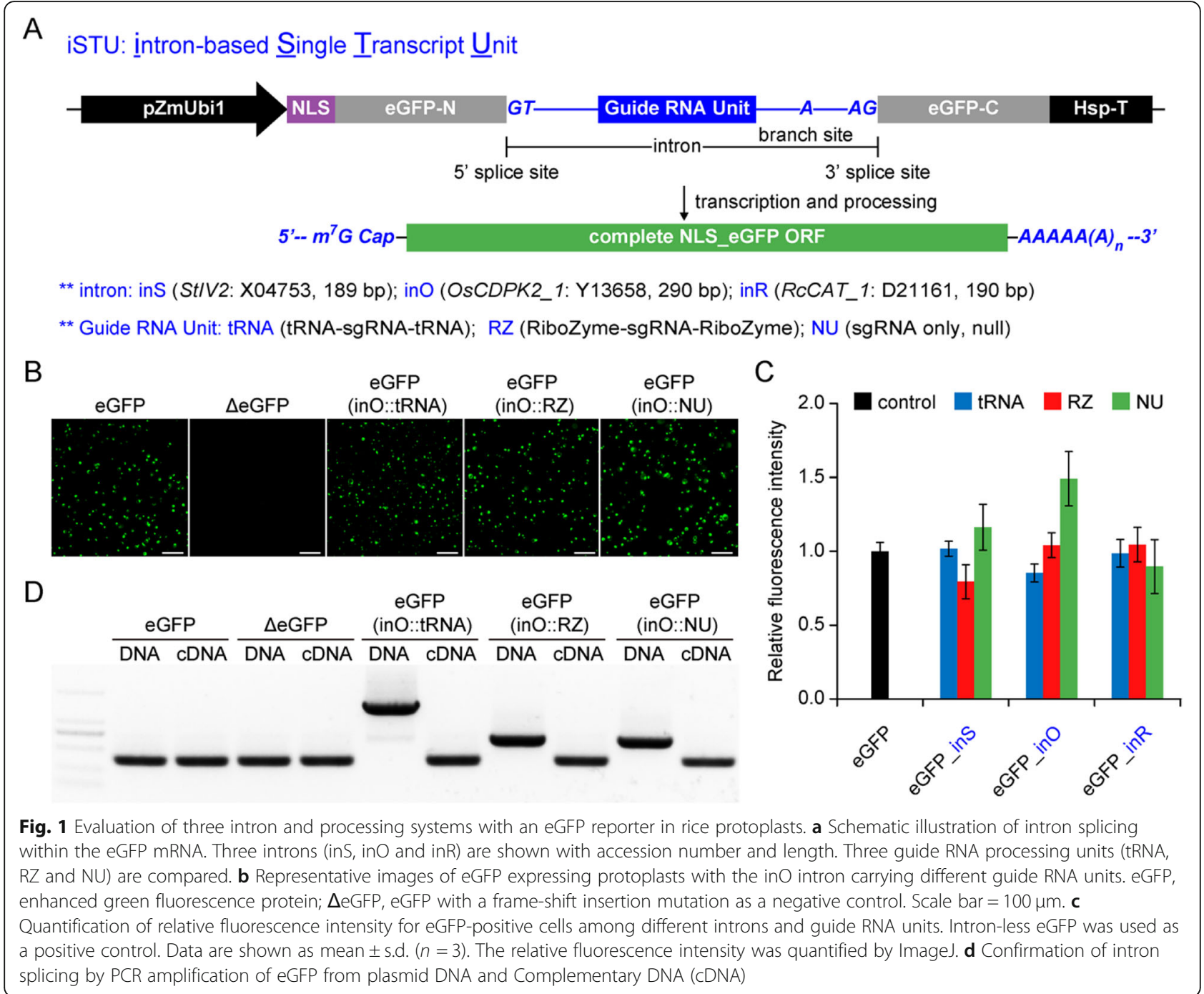

signal to the intro-less vector, regardless the sgRNA processing cassettes (tRNA, RZ or NU) (Fig. 1c). The data suggest that modified intron-containing eGFP vectors achieved similar level of gene expression and efficient splicing of the primary eGFP messenger RNA (mRNA). Focusing on the OsCDPK2_1 intron (inO) system, we used PCR and reverse transcription PCR (RT-PCR) to further validate correct splicing products. Indeed, corrected complementary DNA (cDNA) was detected with expected length, shorter than the PCR products with introns amplified from the vector DNA (Fig. 1d). Precise splicing was further validated by Sanger sequencing of cDNA from all nine intron configurations (Additional file 1: Figure S2), suggesting correct joining of two exons after splicing. This phenotypic and molecular evidence collectively suggests that sgRNA-containing introns can be inserted into the ORF of the gene of interest, such as eGFP, without interfering of splicing.
Comparison of Three sgRNA Processing Systems for iSTUCRISPR/Cas9 Mediated Genome Editing in Rice Protoplasts To determine these spliced introns can carry functional sgRNAs that mediate Cas9 genome editing, we cloned functional protospacer sequences into the sgRNA scaffolds and inserted such modified introns into the ORF of SpCas9 from Streptococcus pyogenes (Fig. 2a). It was anticipated that these introns would be properly spliced to result in mature mRNA of Cas9 for protein translation. Also, the spliced sgRNA-containing introns would be properly processed to generate mature sgRNA, and subsequently enable genome editing when the sgRNA form complex with Cas9. We termed this system as intronbased single transcript unit CRISPR/Cas9 (iSTU-CRISPR/ Cas9) systems (Fig. 2a). To test iSTU-CRISPR/Cas9, we targeted three independent sites in the rice genome with three sgRNAs, OsDEP1-sgRNA01, OsDEP1-sgRNA02 and OsPDS-sgRNA02. We generated 27 iSTU-CRISPR/Cas9 T-DNA vectors with all possible combination of three 


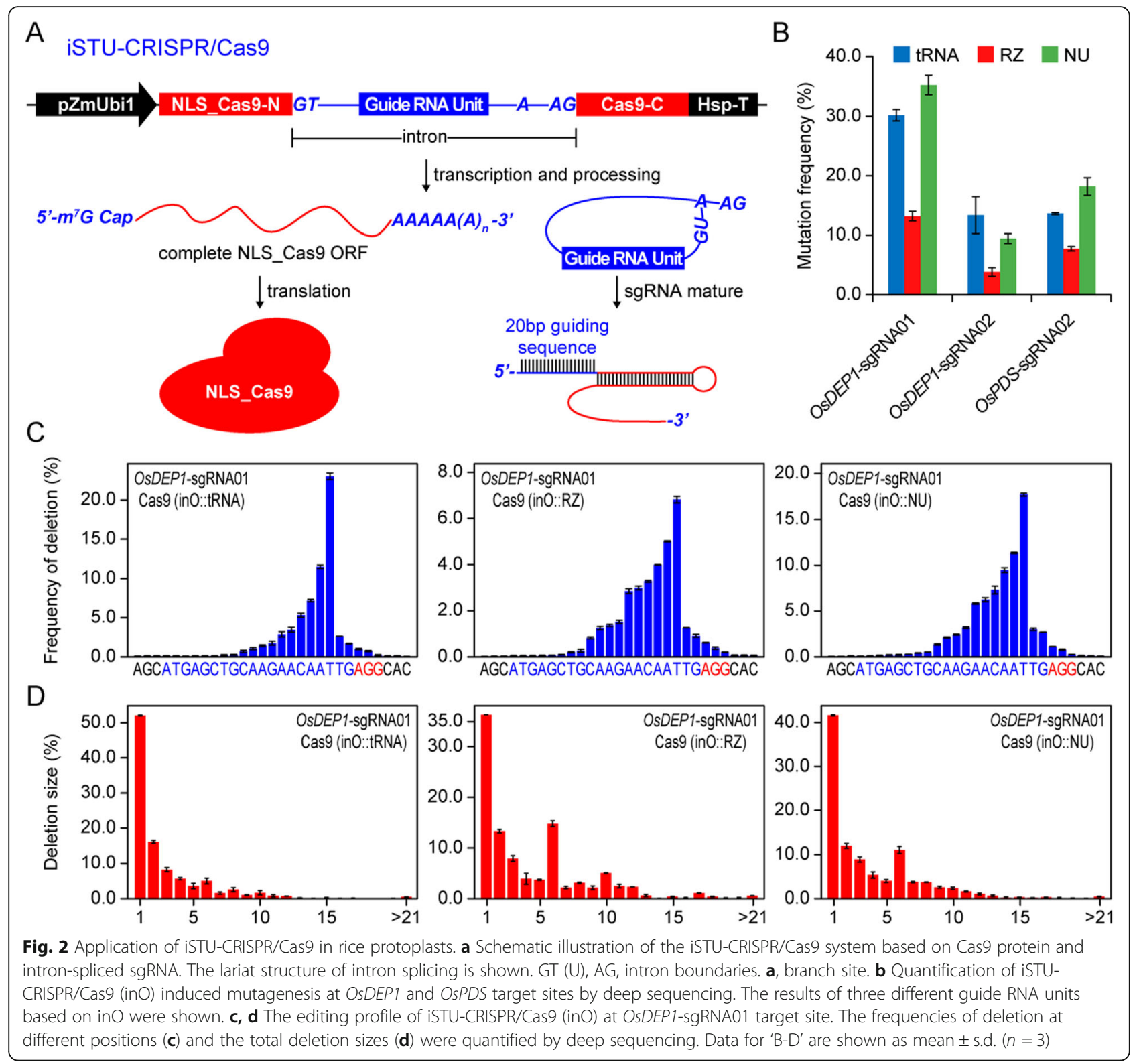

intron systems (inS, inO and inR) and three sgRNA processing systems (NU, tRNA and RZ) at three target sites. We transfected these constructs into rice protoplasts. The results based on restriction fragment length polymorphism (RFLP) showed these constructs all worked in generating targeted insertions and deletions (InDels), which destroyed the restriction enzymes sites of MunI, HhaI and HindIII at the three target sites (Additional file 1: Figure S3). Given that the OsCDPK2_1 intron is derived from the rice genome, we decided to subsequently focus on this intron system (inO) in the study. We used next-generation sequencing (NGS) of PCR amplicons to quantify NHEJ mutation frequencies. The results suggested both the tRNA and NU systems led to robust editing, with efficiencies in rice protoplasts over
$10 \%$ across all three target sites (Fig. 2b). By contrast, the RZ system had relatively poor activity; mutation frequencies were about half compared to the tRNA or the NU system (Fig. 2b).

We next analyzed the NGS data to reveal mutation profiles. Analysis of the results with OsDEP1-sgRNA01 showed that the frequencies of deletions at different positions were very similar among all three sgRNA processing systems for the target site of OsDEP1-sgRNA01 (Fig. 2c), with 1-bp deletions as the most frequent mutations (Fig. 2d). Analysis of the mutation profiles at OsDEP1-sgRNA02 and OsPDSsgRNA02 sites (Additional file 1: Figure S4) further reinforced these findings. In general, the deletion profiles are very similar to our previous reports with the conventional mixed dual promoter Cas9 system (Tang et al. 2018), the 
single transcript unit systems (Tang et al. 2019, Tang et al. 2016), or the bidirectional promoter systems (Ren et al. 2019). The results suggest that NHEJ outcomes by Cas9 are largely reproducible and independent of the CRISPR/Cas9 expression systems.

To investigate whether the iSTU-CRISPR/Cas9 system can be used for multiplexed genome editing, we chose the tRNA system and four genomic sites (OsPDS-sgRNA01, OsPDS-sgRNA02, OsDEP1-sgRNA01 and OsDEP1-sgR NA02). We targeted these sites two a time with four combinations: OsPDS-sgRNA01 and OsPDS-sgRNA02, OsPDS-sgRNA01 and OsDEP1-sgRNA02, OsDEP1-sgR NA01 and OsDEP1sgRNA02, and OsDEP1-sgRNA01 and OsPDS-sgRNA02 (Fig. 3a). The multiplexed T-DNA vectors were generated and transfected into rice protoplasts. Analysis by RFLP showed editing efficiencies at these target sites ranged from $18.6 \%$ to $65.3 \%$ (Fig. 3b). These data demonstrated multiplexed editing in protoplasts by the inO::tRNA based iSTU-CRISPR/Cas9 system.

\section{Comparison of iSTU-CRISPR/Cas9 Genome Editing Systems in Stable Transgenic Lines}

We next conducted rice stable transgenesis to further compare multiple iSTU-CRISPR/Cas9 genome editing systems. To this end, we transformed rice calli with 12 T-DNA vectors corresponding to three sgRNA processing systems (inO::tRNA, inO::RZ and inO::NU) at four target sites: OsDEP1-sgRNA01，OsDEP1-sgRNA02，OsPDS-sgRNA01 and OsPDS-sgRNA02. Up to 50 transgenic T0 lines were obtained for each construct and these T0 lines were genotyped by Sanger sequencing followed by decoding. The

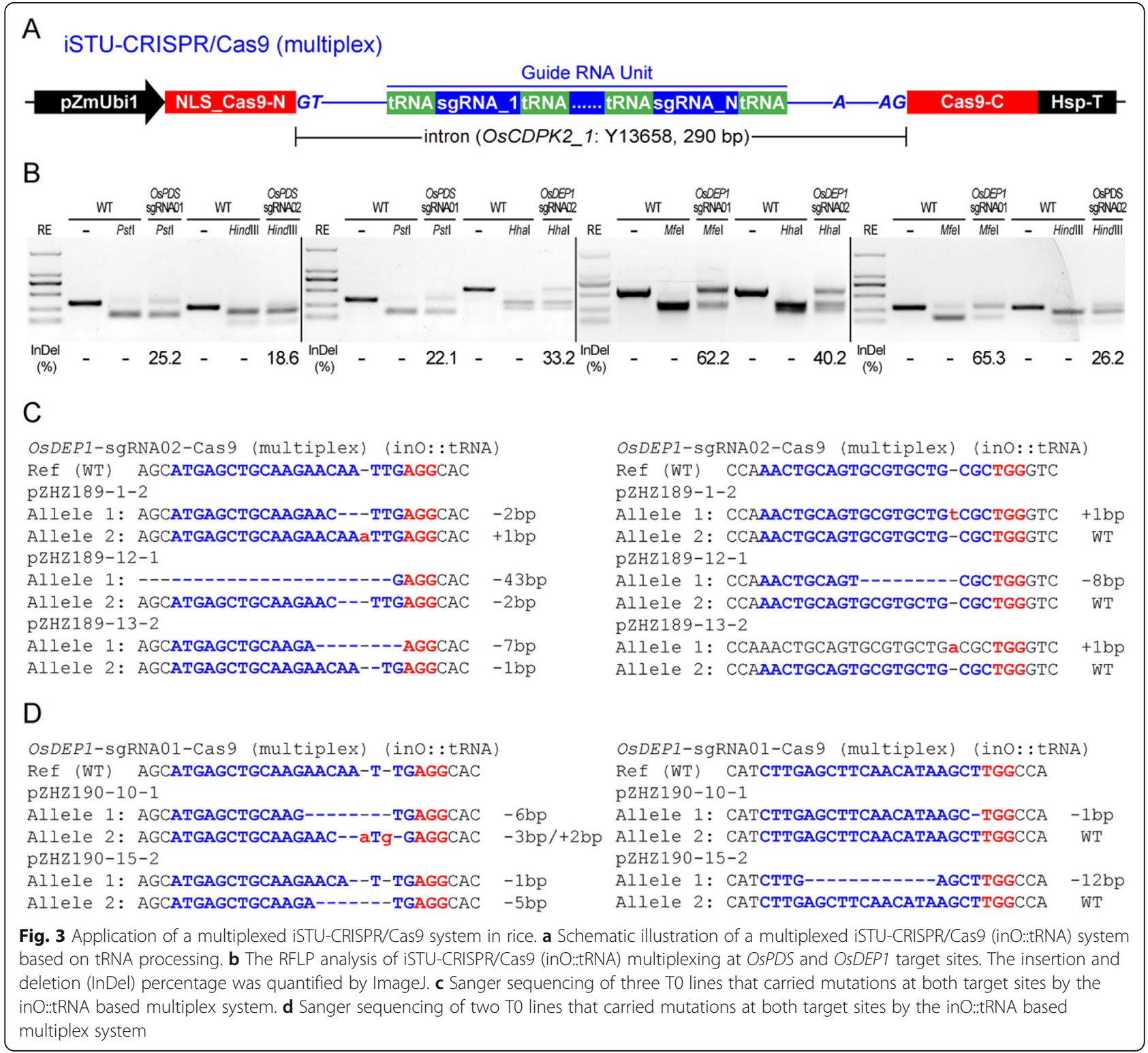


results suggested high editing efficiency with OsDEP1sgRNA01 (as high as 90\% with the inO::tRNA system) and relatively lower efficiencies at the other three target sites (Table 1), which is consistent with the data from protoplasts (Fig. 2b and Fig. 3). Overall, the inO::tRNA system outperformed the inO::RZ and inO::NU systems. Surprisingly, the inO::RZ system showed equal or better editing efficiencies over the inO::NU system. For example, the inO::tRNA system resulted in editing efficiencies of $71.4 \%$ and $84.6 \%$ at OsDEP1-sgRNA01 and OsPDS-sgRNA01 sites, higher than the inO::NU system which only had efficiencies of $44.0 \%$ and $27.3 \%$ at these two sites, respectively (Table 1). Both monoallelic and biallelic mutants were generated by all three iSTU-Cas9 systems at the target sites (Table 1 and Additional files 1: Figures S5-S7).

We also pursued multiplexed editing in rice stable transgenesis with the inO::tRNA based iSTU-CRISPR/Cas9 system. We observed robust editing at the first site, OsDEP1-sgRNA01, with editing efficiencies of $58.6 \%$ and $71.4 \%$ by two constructs respectively. However, we only observed low editing efficiency at the second site either with OsDEP1-sgRNA02 (10.0\% editing efficiency) or OsPDSsgRNA02 (4.0\% editing efficiency) (Table 1). Regardless, we were successful in identifying multiple T0 lines that carried mutations at both target sites (Fig. 3c and d).

\section{Development of iSTU-CRISPR/Cas12a Systems in Genome Editing in Rice Protoplasts}

We reasoned that our intron systems could also be used to express crRNAs for CRISPR-Cas12a mediated genome editing. There are two popular systems for processing
crRNAs to maturity in plants, one based on self-cleavage of direct repeat (DR) by Cas12a's intrinsic RNase activity (Fonfara et al. 2016, Zetsche et al. 2017) and the other based on a dual HH-HDV ribozyme system (Tang et al. 2017). We positioned the DR-crRNA-DR or the HHcrRNA-HDV cassette in between the $5^{\prime}$ splice site and the branch site of the OsCDPK2_1 intron, and the modified intron with a crRNA unit was inserted into the ORF of a rice codon-optimized LbCas12a from Lachnospiraceae bacterium ND2006 (Tang et al. 2017) (Fig. 4a). To compare these two iSTU-CRISPR/Cas12a systems, we targeted three rice genomic sites with OsDEP1-crRNA01, OsDEP1crRNA02 and OsROC5-crRNA02. The resulting six expression vectors were used for rice protoplast transfection. Targeted mutations at these three sites were evaluated by RFLP, which showed detectable mutations at all three target sites (Additional file 1: Figure S8). We then used NGS to further quantify mutation frequencies at the target sites. The DR-DR system showed higher editing efficiencies than the HH-HDV system at OsDEP1-crRNA01 and OsDEP1-crRNA02 sites, while both systems showed equivalent editing efficiencies at OsROC5-crRNA02 site (Fig. 4b). These data indicate that the DR-DR system is more robust than the HH-HDV system when coupled with the intron expression system.

We analyzed the NGS data for deletion profiles by the iSTU-CRISPR/Cas12a systems. The DR-DR system resulted in deletions that mostly affected the nucleotides close to the cleavage sites and the deletions were much larger than Cas9 induced deletions (Fig. 4c and d). The same deletion profiles were revealed by the HH-HDV

Table 1 Genome editing in stable transgenic rice lines with intron based Cas9 system

\begin{tabular}{|c|c|c|c|c|c|}
\hline Targeted rice sites & CRISPR scaffold & Tested T0 lines & $\begin{array}{l}\text { Mutated T0 lines } \\
\text { (number; ratio) }\end{array}$ & $\begin{array}{l}\text { Biallelic mutation lines } \\
\text { (number; ratio) }\end{array}$ & Constructs \\
\hline OsDEP1-sgRNA01 & \multirow[t]{4}{*}{ Cas9 (inO::tRNA) } & 10 & $9,90.0 \%$ & $7,70.0 \%$ & pZHZ159 \\
\hline OsDEP1-sgRNA02 & & 12 & $5,46.5 \%$ & $2,16.7 \%$ & $\mathrm{pZHZ160}$ \\
\hline OsPDS-sgRNA01 & & 15 & $9,60.0 \%$ & $2,13.3 \%$ & pZHZ161 \\
\hline OsPDS-sgRNA02 & & 26 & $14,53.8 \%$ & $6,23.1 \%$ & pZHZ162 \\
\hline OsDEP1-sgRNA01 & \multirow[t]{4}{*}{ Cas9 (inO::RZ) } & 14 & $10,71.4 \%$ & $4,28.6 \%$ & pZHZ167 \\
\hline OsDEP1-sgRNA02 & & 6 & $2,33.3 \%$ & $1,16,7 \%$ & pZHZ168 \\
\hline OsPDS-sgRNA01 & & 18 & $11,84.6 \%$ & $3,16,7 \%$ & pZHZ169 \\
\hline OsPDS-sgRNA02 & & 10 & $1,10.0 \%$ & $1,10.0 \%$ & pZHZ170 \\
\hline OsDEP1-sgRNA01 & \multirow[t]{4}{*}{ Cas9 (inO::NU) } & 25 & $11,44.0 \%$ & $8,32.0 \%$ & pZHZ171 \\
\hline OsDEP1-sgRNA02 & & 12 & $4,33.3 \%$ & $0,0.0 \%$ & pZHZ172 \\
\hline OsPDS-sgRNA01 & & 11 & $3,27.3 \%$ & $2,16.7 \%$ & pZHZ173 \\
\hline OsPDS-sgRNA02 & & 37 & $11,29.7 \%$ & $1,9.1 \%$ & pZHZ174 \\
\hline OsDEP1-sgRNA01 & \multirow[t]{2}{*}{ Cas9 (inO::tRNA) } & 30 & $17,58.6 \%$ & $7,23.3 \%$ & \multirow{2}{*}{$\begin{array}{l}\text { pZHZ189 } \\
\text { (Multiplex) }\end{array}$} \\
\hline OsDEP1-sgRNA02 & & 30 & $3,10.0 \%$ & $0,0.0 \%$ & \\
\hline OsDEP1-sgRNA01 & \multirow[t]{2}{*}{ Cas9 (inO::tRNA) } & 50 & $30,71.4 \%$ & $20,40.0 \%$ & \multirow{2}{*}{$\begin{array}{l}\text { pZHZ190 } \\
\text { (Multiplex) }\end{array}$} \\
\hline OsPDS-sgRNA02 & & 50 & $2,4.0 \%$ & $0,0.0 \%$ & \\
\hline
\end{tabular}




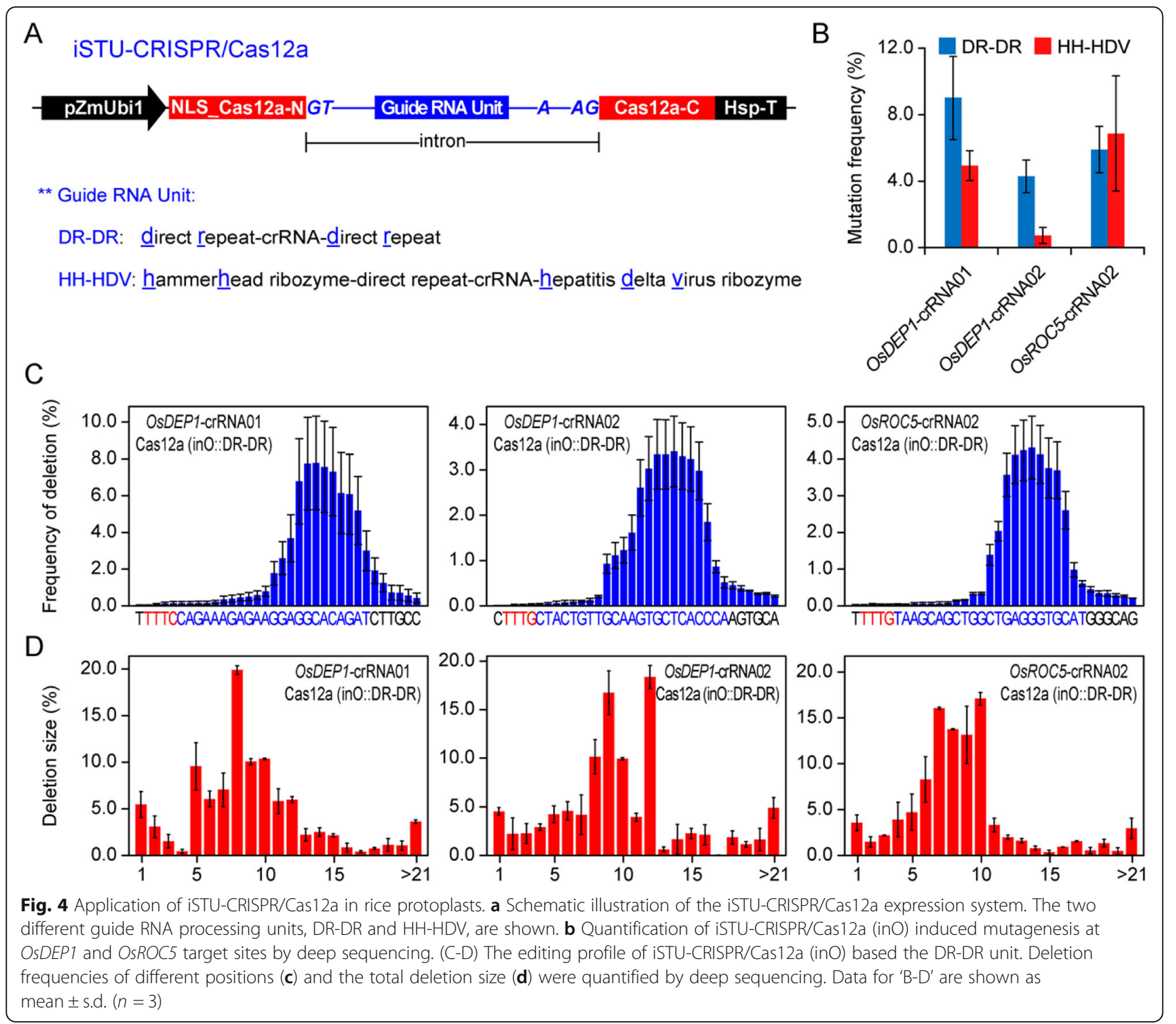

system (Additional file 1: Figure S9). These data reinforce our previous conclusions regarding Cas12a induced large deletions in plants (Malzahn et al. 2019, Tang et al. 2018, Tang et al. 2017, Tang et al. 2019, Zhong et al. 2018), which support that overall mutation profiles by Cas12a are also reproducible within a large cell population and such profiles are not affected by the expression systems.

We next used DR-DR based CRISPR array and an HHHDV array to multiplex two crRNAs with the iSTUCRISPR/Cas12a system (Fig. 5a). We simultaneously expressed two crRNAs for deletion of a DNA fragment of 289 bp in OsDEP1 and used another two crRNAs for deletion of a DNA fragment of $295 \mathrm{bp}$ in OsROC5. Two multiplexed Cas12a constructs were generated for rice protoplast transfection. For the DR-DR samples, we detected chromosomal deletions by PCR at both genes with frequencies of $4.5 \%$ and $6 \%$, respectively, and the large deletions were further validated by Sanger sequencing (Fig. 5b). However, we could not detect deletion events at either gene with PCR for the HH-HDV system (Fig. 5b). The data suggest the DRDR based CRISPR array, not the HH-HDV array, is more suitable for intron-based processing that enables Cas12a mediated multiplexed genome editing. This observation is consistent with our earlier findings regarding iSTUCRISPR/Cas12a system with singular crRNAs (Fig. 4b).

\section{Evaluation of iSTU-CRISPR/Cas12a Systems for Genome Editing in Stable Transgenic Lines}

To assess whether we can obtain mutants with the iSTU-CRISPR/Cas12a systems, we compared the DR-DR and HH-HDV systems in stable rice transformants (Table 2). With OsDEP1-crRNA01, the DR-DR system generated 7 mutants out of 15 T0 lines (46.7\%) and the HH-HDR only generated one mutant out of 19 T0 lines 


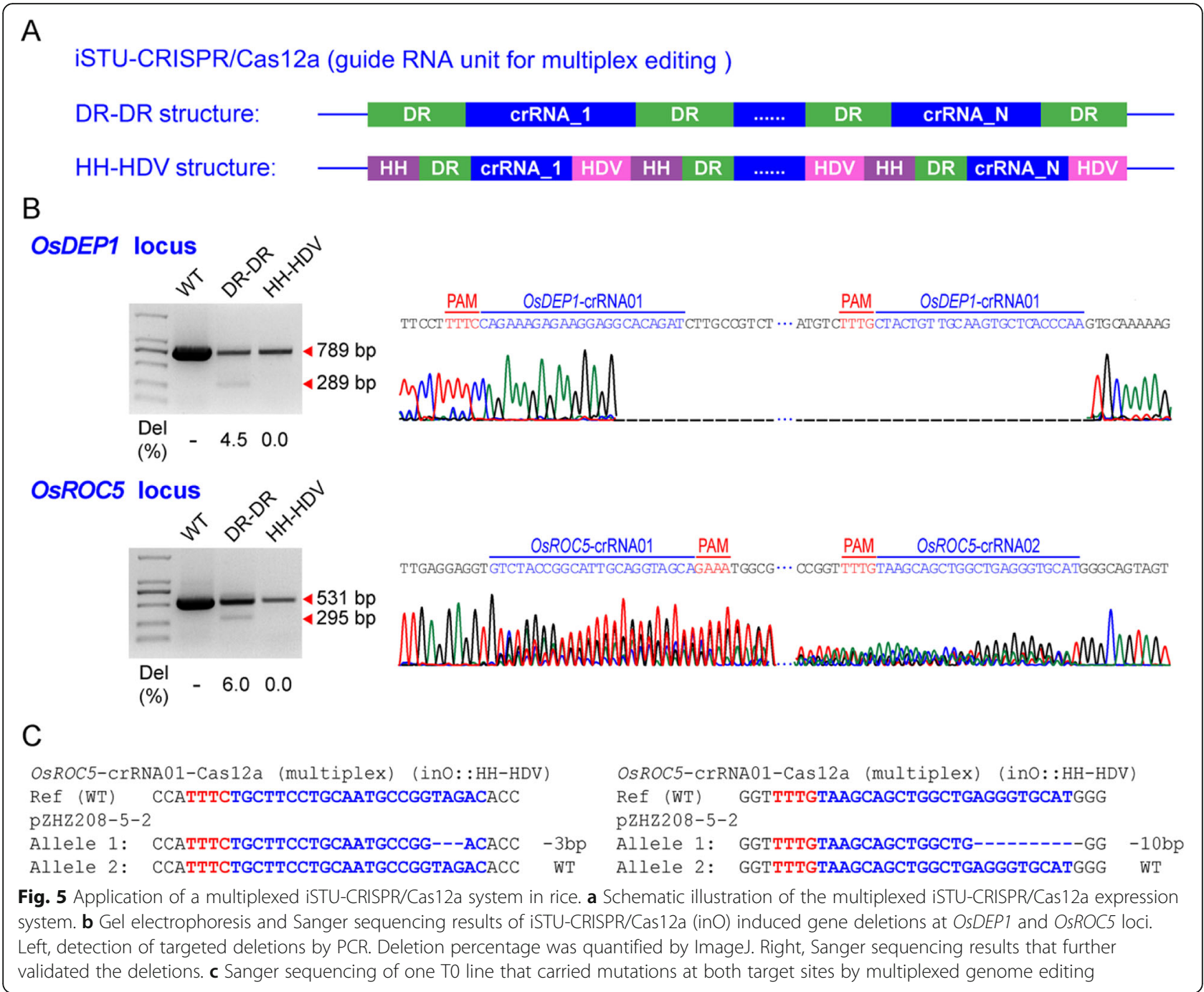

(5.3\%). With OsROC5-crRNA01, the DR-DR system generated 2 mutants out of 22 T0 lines (9.1\%) and the HHHDV failed to generate any mutant out of 21 T0 lines. These data suggest the DR-DR system is more robust than the HH-HDV system for iSTU-CRISPR/Cas12a mediated targeted mutagenesis in stable rice lines, consistent with our observations in rice protoplasts.

In rice protoplasts, the HH-HDV based multiplex system did not generate detectable large deletions at both target genes when assayed by PCR (Fig. 5b), which may be largely due to its low editing efficacy. This system however may be able to introduce targeted mutations at individual sites. We investigated this possibility with rice stable transgenesis using the multiplexed HH-HDV construct targeting OsROC5. Among 22 T0 lines assayed, one line showed mutation by OsROC5-crRNA01 and three lines had mutations by OsROC5-crRNA02, resulting mutation frequencies of $4.5 \%$ and $13.7 \%$, respectively (Table 2 ). Notably, one line (pZHZ208-5-2) had mutations in both target genes (Fig. 5c). The data suggest the multiplexed $\mathrm{HH}$ HDV array, when configured into the iSTU system, can generated rice mutants at individual target sites, albeit at a low efficiency.

\section{Discussion}

Since many plant genes contain introns, it is intriguing to configure a transgene such as Cas9 or Cas12a that contains introns. First, it is well known that some introns can boost gene expression (Jeong et al. 2006). The plant codon optimized Cas9 (pcoCas9) contains an intron (Li et al. 2013), which may prevent the Cas9 transgene from gene silencing across multiple generations. We hence adopted pcoCas9 for engineering synthetic transcriptional repressors and activators (Lowder et al. 2015, Lowder et al. 2018). Second, adding introns to the transgene can abolish potential toxicity in bacteria (e.g. E. coli) during the molecular cloning process. For example, we previously introduced an intron into the ORF 
Table 2 Genome editing in stable transgenic rice lines with intron based Cas12a system

\begin{tabular}{|c|c|c|c|c|c|}
\hline Targeted rice sites & CRISPR scaffold & Tested T0 lines & $\begin{array}{l}\text { Mutated T0 lines } \\
\text { (number; ratio) }\end{array}$ & $\begin{array}{l}\text { Biallelic mutation lines } \\
\text { (number; ratio) }\end{array}$ & Constructs \\
\hline OsDEP1-crRNA01 & \multirow[t]{2}{*}{ Cas12a (inO::DR-DR) } & 15 & $7,46,7 \%$ & $0,0.0 \%$ & $\mathrm{pZHZ144}$ \\
\hline OsROC5-crRNA-01 & & 22 & $2,9.1 \%$ & $0,0.0 \%$ & $\mathrm{pZHZ146}$ \\
\hline OsDEP1-crRNA01 & \multirow[t]{2}{*}{ Cas12a (inO:: HH-HDV) } & 19 & $1,5.3 \%$ & $0,0.0 \%$ & pZHZ148 \\
\hline OsROC5-crRNA-01 & & 21 & $0,0.0 \%$ & $0,0.0 \%$ & pZHZ150 \\
\hline OsROC5-crRNA01 & \multirow[t]{2}{*}{ Cas12a (inO:: $\mathrm{HH}-\mathrm{HDV}$ ) } & 22 & $1,4.5 \%$ & $0,0.0 \%$ & \multirow{2}{*}{$\begin{array}{l}\text { pZHZ208 } \\
\text { (Multiplex) }\end{array}$} \\
\hline OsROC5-crRNA02 & & 22 & $3,13.7 \%$ & $0,0.0 \%$ & \\
\hline
\end{tabular}

of TAL effector nuclease (TALEN) to avoid toxicity in $E$. coli and Agrobacterium (Christian et al. 2013). Third, insertion of an gRNA-containing intron to the ORF of Cas9 or Cas12a represents a more authentic gene structure for expression of both the Cas gene and the gRNA in a single mRNA. It is thus imperative to the development of novel intron-based STU CRISPR genome editing systems. With appropriate intron choices and proper positioning in the Cas gene, one would configure a novel STU CRISPR-Cas system that mimics an endogenous gene structure. Previously, Ding et al. explored a 5' UTR intron to express sgRNA or crRNA for Cas9 or Cas12a mediated genome editing in rice (Ding et al. 2018). Compared to the $5^{\prime}$ UTR, there are numerous positions in the ORFs of Cas9 and Cas12a for intron insertions. The only previous study that employed an intron to express an sgRNA within a Cas9 for plant genome editing was done in Chlamydomonas reinhardtii, which showed very low editing efficiency likely due to toxicity of Cas9 in this green algae (Jiang \& Weeks 2017). In this study, we explored multiple iSTU systems where gRNAcontaining introns were inserted in the middle of a Cas9 or Cas12a ORF. We tested three introns from three plant species, including an StIV2 intron from potato, an OsCDPK2_1 intron from rice and a $R c C A T \_1$ intron from castor bean. Using an eGFP reporter system, we first demonstrated these introns, when carrying gRNA units, could be properly spliced in rice. We then applied these introns to carry functional sgRNA or crRNA cassettes and successfully showed genome editing by either Cas9 or Cas12a, in both protoplasts and stable transgenic rice lines. Our success suggests many different introns may be amendable to carry gRNAs for CRISPR genome editing in plants.

For detailed assessment of our iSTU systems, we later focused on the OsCDPK2_1 intron (e.g. inO) as it is an endogenous intron from the rice genome. With the inOCas9 system, we compared insertion of sgRNAs with and without added processing mechanisms. We found tRNA based processing is most robust for genome editing in both protoplasts and stable transgenic lines. The processing based on a single $\mathrm{HH}$ ribozyme is less efficient. These observations are consistent with our recent findings when developing CRISPR-STU2.0 systems (Tang et al. 2019). Interestingly, we also found the iSTU system without any added processing mechanism also worked, which showed equivalent editing efficiencies to the $\mathrm{HH}$ ribozyme systems. This result is consistent with previous findings (Mikami et al. 2017, Wang et al. 2018), indicating possible sgRNA maturation based on an unknown RNase in rice. With the inO-Cas12a system, we compared two crRNA processing mechanisms, DR-DR and HH-HDV. The DR-DR inO-Cas12a system showed much better results than the one based on HH-HDV. Our most efficient Cas12a system is a dual Pol II promoter system which relies on HH-HDV for crRNA processing (Tang et al. 2017, Zhong et al. 2018). The contrasting efficiencies of the HH-HDV processing strategies in different expression systems suggest the selfcleavage feature of $\mathrm{HH}$ and HDV ribozymes may not be compatible with the intron system. Although we did not find abnormal splicing with an HH-ribozyme carrying intron in the eGFP reporter system, it is possible the instant self-cleavage by $\mathrm{HH}$ and $\mathrm{HDV}$ ribozymes negatively affected intron splicing or crRNA maturation, which can explain the observed low editing efficiency with the HH-HDV based iSTU-Cas12a system.

The overall genome editing efficiency of our iSTU systems, either with Cas9 or Cas12a, is similar in some degree to 5' UTR intron systems previously reported (Ding et al. 2018). Compared to the 5' UTR intron systems, our iSTU systems are promoter-independent and should be readily applicable to other plant species. At this point, these intron-based CRISPR systems seem sub-optimal in terms of genome editing efficiency when compared to other STU systems where gRNA cassettes were positioned at the 3' end (Tang et al. 2019, Tang et al. 2016, Wang et al. 2018). We noted our iSTU systems became less efficient when more than one gRNA was inserted into the intron, making our systems less robust for multiplexed genome editing. This may be explained by the use of relatively small introns as all three introns we used were under $300 \mathrm{bp}$. It is likely that insertion of multiple gRNA cassettes into these small introns significantly affect the intron structure. It is thus worthwhile to test larger introns in future investigations which 
may improve the overall genome editing efficiency of the iSTU systems. Alternatively, it may be more effective to use multiple introns to individually carry each gRNA cassette for developing multiplexed iSTU systems. Based on our data with singular gRNA and multiple gRNAs, it appears such a multi-intron strategy may work better and hence is worth future exploration.

While splicing sites can be designed and validated relatively easily, the fate of the introns after splicing is largely unknown. It is also unknown whether insertion of gRNA cassettes into an intron will affect its turnover rate. Given Cas9 and Cas12a each has an ORF well over $3 \mathrm{~kb}$, there are many positions within the ORF for intron insertion. In this study, we only arbitrarily chose one site in Cas9 or Cas12a for intron insertion. It is hard to believe the position that we chose is the ideal position. While many intron insertion sites may be tested, we believe the choice of introns and the choice of gRNA insertion site are both key to enhanced editing efficiency. While the focus of this study is to develop iSTU systems for plant genome editing, we envision such iSTU systems may be useful for CRISPR based transcriptional regulation. The iSTU systems may also allow for indirect measurement of splicing efficiency for certain introns as well as for understanding the fate of introns after splicing.

\section{Conclusion}

With rice as a test platform, we developed multiple iSTU systems (STU CRISPR 3.0) for Cas9 and Cas12a mediated genome editing in plants. While the genome editing efficiency is still sub-optimal, this proof-of-concept study has shed light on improved iSTU systems in plants.

\section{Materials and Methods Vector Construction}

The eGFP-intron expression, iSTU-CRISPR/Cas9 and iSTU-CRISPR/Cas12a backbone vectors in this study were constructed by Gibson Assembly ${ }^{\circ}$ Cloning Kit from New England Biolabs (Ipswich, MA, USA). Plasmid was isolated using Axygen Mini Plasmid Kit (Corning, NY, USA). Gel extraction was conducted using AxyPrep DNA Gel Extraction Kit (Corning, NY, USA). Oligonucleotides were synthesized by Sangon Biotech (Shanghai, $\mathrm{CHN}$ ). The DNA fragments were synthesized by IDT (Integrated DNA Technologies, IA, USA). The fast digest enzymes were purchased from Thermo Fisher Scientific (Waltham, MA, USA). All other reagents used here were purchased from New England Biolabs (Ipswich, MA, USA). All the oligos were summarized in Additional file 2: Table S1.

To generate the eGFP-intron expression backbone, three introns with three guide RNA unit structures (NU, tRNA, RZ) were synthesized. To generate pZHZ113 for the eGFP expression, eGFP was amplified with primers
ZHZ113-A and ZHZ113-B from pZHY981. The tHSP part was amplified from pZHY988 with primers ZHZ113-C and ZHZ113-D. Both PCR fragments were assembled with SdaI and HindIII digested pZHZ127 plasmid by Gibson Assembly. The final vector was sequenced by ZY065-RB and ZmUbi-Seq for confirmation. To generate pZHZ114 for the $\triangle \mathrm{eGFP}$ expression, the eGFP fragment 1 with $\mathrm{BsaI}$ insertion was PCR amplified with primers ZHZ113-A and ZHZ113-E from pZHZ113; the eGFP fragment 2 with $B s a \mathrm{I}$ insertion and tHSP part were PCR amplified with ZHZ113$\mathrm{F}$ and ZHZ113-D from pZHZ113; the three parts were fused with primers ZHZ113-A and ZHZ113-D by fusion PCR. The final vector was generated by Gibson Assembly cloned into SdaI and HindIII digested pZHZ113 plasmid and sequenced by ZY065-RB and ZmUbi-Seq for confirmation. The resulting $\triangle \mathrm{eGFP}$ was frame-shifted with a $16 \mathrm{bp}$ insertion. To generate pZHZ118-pZHZ126 eGFP expression vectors with different introns and different guide RNA unit structures, nine DNA fragments were synthesized by IDT. The diluted fragments were cloned into BsaI digested pZHZ114 plasmid by Gibson Assembly. The final vector was sequenced by ZY065-RB and ZmUbi-Seq for confirmation. Notably, the tRNA based guide RNA structure contained a $c c d B$ gene insertion.

To generate iSTU-Cas9 expression vector with different intron and different guide RNA unit insertions, the Cas9 fragment 1 with $B s a I$ insertion was PCR amplified with primers ZHZ113-A and ZHZ112-B. The Cas9 fragment 2 and tHSP part were amplified with primers ZHZ112-C and ZHZ113-D. These two fragments were fused together by fusion PCR with primers ZHZ113-A and ZHZ113-D. The fused fragment was cloned into pZHZ127 by Gibson Assembly to make pZHZ128. To generate iSTU-CRISPR/Cas9 backbone vectors, pGEL077 to pGEL086, nine fragments were amplified from pZHZ118 to pZHZ126 with specific primer sets (Additional file 2: Table S1), and then cloned into BsaI digested pZHZ128 plasmid by Gibson Assembly. The vector was confirmed by Sanger sequencing using primers dCas9-F and dCas9-R.

To generate iSTU-CRSPR/Cas12a backbone vector pGEL087, oligos ZHZ141-F and ZHZ141-R were annealed and ligated into SpeI and AscI sites of pTX377 to remove the crRNA expression cassette, resulting in pGEL087Step1. The LbCas12a part I was PCR amplified from pTX377 with primers ZHZ141-F1 and ZHZ141-R1. The LbCas12a part II was amplified from pTX377 with primers ZHZ141-F2 and ZHZ141-R2. The two parts were fused via PCR with primers ZHZ141-F1 and ZHZ141-R2, and the resulting PCR fragment was cloned into BglII and SphI sites of pGEL087-Step1 plasmid. To generate iSTUCRISPR/Cas12a vector, pGEL088 and pGEL089, the synthesized fragments were cloned into $B s a \mathrm{I}$ digested pGEL087 by Gibson Assembly. 
To generate the T-DNA vector, the oligos were synthesized based on the target sites selected. The oligo pair for each guide RNA was annealed and inserted into BsaI sites of the backbone vectors by Golden Gate cloning according our published protocol (Lowder et al. 2015). To generate iSTU-CRISPR/Cas9 and iSTU-CRISPR/Cas12a multiplex T-DNA vectors, the synthesized fragments were cloned into corresponding backbones by Golden Gate cloning. These T-DNA expression vectors (Additional file 3: Table S2) were confirmed by RFLP and Sanger Sequencing.

\section{Plant Material and Growth Condition}

The Japonica cultivar Nipponbare was used in this study. For protoplast preparation, the sterilized seeds were germinated in the $1 / 2$ MS solid medium for 11 days in dark chamber at $28{ }^{\circ} \mathrm{C}$. For the rice stable transformation, the sterilized seeds were germinated in the N6-D solid medium to induce the callus for 7 days in light at $32^{\circ} \mathrm{C}$.

\section{Rice Protoplast Preparation, Transformation and Analysis}

The rice protoplast extraction and transformation method was performed according to our previously published protocol (Tang et al. 2016, Zhong et al. 2018). Leaves of rice seedlings were cut into $0.5-1.0 \mathrm{~mm}$ strips, and transferred into the enzyme solution followed by vacuum-infiltration for $30 \mathrm{~min}$. The mixture was gently agitated at $70-80 \mathrm{rpm}$ for $8 \mathrm{~h}$ at $25^{\circ} \mathrm{C}$ in the dark. The digestion mixture was filtered by a $40 \mu \mathrm{m}$ cell strainer. After washing the protoplasts two times with W5 washing buffer, cell counts of protoplasts was done using a hemacytometer under a microscope and the final protoplast concentration was adjust to $2 \times 10^{6}$ per milliliter. For protoplast transformation, $30 \mu \mathrm{L}$ plasmid DNA ( $1 \mu \mathrm{g} / \mu \mathrm{L}$; prepared by Axygen midiprep kit) was used for transformation of $200 \mu \mathrm{L}$ protoplasts by gently mixing with $230 \mu \mathrm{L} 40 \%$ PEG transformation buffer. After incubating $30 \mathrm{~min}$ in the dark, the reaction was terminated by adding $1 \mathrm{~mL} \mathrm{W5}$ washing buffer. The protoplasts were centrifuged at $250 \times \mathrm{g}$ and transferred into a 12-well culture plate at $32^{\circ} \mathrm{C}$ in the dark. After 2 days' incubation, images of the cells were taken by a fluorescence microscope with a GFP filter or DNA was extracted from the cells using the CTAB method (Murray \& Thompson. 1980). The RFLP method was used to detect NHEJ mutations induced by Cas9 or Cas12a at the target sites. The PCR was performed using Tsingke Golden Mix (Tsingke, Beijing) with specific primers (see Additional file 2: Table S1). The PCR products were digested overnight and resolved by electrophoresis in $1 \%$ TAE agarose gels.

\section{Rice Stable Transformation and Genotyping}

The Agrobacterium-mediated rice transformation was performed as the protocol we published before (Zhou et al. 2017, Zhou et al. 2019). The calli for stable transformation were cultured in the chamber for 7 days at $32{ }^{\circ} \mathrm{C}$ under light. The binary T-DNA vectors were transferred into Agrobacterium tumefaciens strain EHA105. The transformed Agrobacterium EHA105 cells were cultured in liquid LB medium at $28^{\circ} \mathrm{C}$ for 2 days. The cells were then collected and resuspended in liquid AAM-AS medium (OD600 $=0.1$ ) containing $100 \mu \mathrm{M}$ acetosyringone. After cocultivation of Agrobacteria and calli for 3 days, the calli were washed with sterilized water and transferred to N6-S medium containing $400 \mathrm{mg} / \mathrm{L}$ Carbenicillin and $50 \mathrm{mg} /$ L Hygromycin for 2 weeks. Then they were transferred to REIII medium with $400 \mathrm{mg} / \mathrm{L}$ Carbenicillin and 50 $\mathrm{mg} / \mathrm{L}$ Hygromycin cultured for 2 weeks, and the resistant calli were later transferred to fresh REIII medium for every 2 weeks until the regeneration of T0 plants. To genotype T0 plants, the SSCP method (Zheng et al. 2016) was used for a first round of screen, followed by Sanger sequencing of positive lines.

\section{The NGS Sample Preparation, Detection and Analysis}

Next-generation sequencing (NGS) of PCR amplicons was also used for detection and quantification of mutations present in protoplast samples. With the protoplast DNA as template, the DNA sequence flanking each target site was amplified by using Tsingke Golden Mix Kit (Tsingke, Beijing) and the barcodes were added to the end of the primers. The PCR products were resolved by electrophoresis in 1\% agarose gel for checking purity and concentration. The PCR product were sent to the Novogene and sequenced by using an Illumina Hiseq 2500 platform. The clean data were analysis by CRISPRMatch (You et al. 2018).

\section{Supplementary information}

Supplementary information accompanies this paper at https://doi.org/10. 1186/s12284-020-0369-8.

\footnotetext{
Additional file 1: Figure S1. eGFP expression of ins and inR intron splicing systems in rice protoplasts. Three different guide RNA units were tested, and all showed GFP signals, indicating of correct splicing. Scale bar $=100 \mu \mathrm{m}$. Figure S2. Sanger sequencing confirmation of inS, inO and inR intron-based slicing of eGFP mRNA. The cDNA of three different intron splicing systems was used for PCR amplification with a primer set flanking the introns. All sanger sequencing results showed correct intron splicing compared with intron-less eGFP. The splicing site was indicated by a dotted red line. Figure S3. RFLP based detection of targeted mutagenesis by iSTU-CRISPR/Cas9 in rice protoplasts. Three introns (inS, inO and inR) with three different guide RNA units were tested at OSDEP1 and OSPDS target sites. The restriction enzymes used for RFLP are shown. Uncut bands are indicative of induced mutations by Cas9. Figure S4. Editing profiles of iSTU-CRISPR/Cas9 (inO) at two additional target sites in rice protoplasts. The editing profiles of iSTU-CRISPR/Cas9 (inO) at OSDEP1sgRNA02 and OsPDS-sgRNA02 target sites. Deletion frequencies at different positions (A) and frequencies of deletion sizes (B) were quantified by deep sequencing. Data are shown as mean \pm s.d. $(n=3)$. Figure S5. Examples of T0 rice mutants generated by iSTU-CRISPR/Cas9 (inO::tRNA) at four target sites. Figure S6. Examples of T0 rice mutants generated by iSTU-CRISPR/Cas9 (inO::RZ) at four target sites. Figure S7. Examples of T0 rice mutants generated by iSTU-CRISPR/Cas9 (inO::NU) at
} 
four target sites. Figure S8. RFLP analysis of iSTU-CRISPR/Cas12a (inO) in rice protoplasts. (a). RFLP analysis of two iSTU-CRISPR/Cas12a systems, inO::DR-DR and inO::HH-HDV. Three target sites were tested. (b). RFLP analysis of two multiplexed iSTU-CRISPR/Cas12a systems, inO::DR-DR and inO::HH-HDV. OsDEP1 and OsROC5 locus were tested with each targeted by two crRNAs. 'A-B', the restriction enzymes are shown and the InDel percentages were quantified by ImageJ. Figure S9. Editing profiles of three target sites by iSTU-CRISPR/Cas12a (inO) HH-HDV system in rice protoplasts. The editing profiles of iSTU-CRISPR/Cas12a (inO) HH-HDV system at OsDEP1-crRNA01, OsDEP1-crRNA02 and OsROC5-crRNA02 target sites. Deletion frequencies of different positions (A) and frequencies of deletion sizes (B) were quantified by deep sequencing. Data are shown as mean \pm s.d. $(n=3)$. Figure S10. Examples of T0 rice mutants generated by the iSTU-CRISPR/Cas12a (inO) systems.

Additional file 2: Table S1. Oligos used in this study.

Additional file 3: Table S2. T-DNA constructs used in this study.

\section{Abbreviations}

CRISPR/Cas9 or 12a: Clustered regularly interspaced short palindromic repeats/CRISPR-associated protein-9 or 12a; iSTU: intron-based Single transcript unit; RFLP: Restriction fragment length polymorphism

\section{Acknowledgements}

We thank Aimee Malzahn for proofreading this manuscript.

\section{Authors' Contributions}

YZ proposed the project and designed the experiments. ZZ and SL designed sgRNAs and constructed all the vectors. ZZ and SL conducted rice protoplast assay, ZZ, SL, XL, BL, XT, QR, JZ and XZ did the rice stable transformation. ZZ, $S L, J Z$, and $X Z$ performed NGS data analysis. $Y Z, Y Q, Z Z$ and $S L$ analyzed the data. $Y Q, Y Z$ and $Z Z$ wrote the manuscript. All authors participated in discussion and revision of the manuscript. All authors read and approved the final manuscript.

\section{Funding}

This work was supported by the National Transgenic Major Project (2019ZX08010003-001-002), the National Science Foundation of China (31771486 and 31960423), the Science Strength Promotion Program of UESTC and the Open Foundation of Jiangsu Key Laboratory of Crop Genomics and Molecular Breeding (PL201801) and the Science Strength Promotion Program of UESTC to Y. Z. and X. Z., and the National Science Foundation Plant Genome Research Program (IOS-1758745), Foundation for Food and Agriculture Research (593603), and a Syngenta Biotechnology Research Grant to Y.Q.

\section{Availability of Data and Materials}

All data generated were presented in the article and included in the Additional files. The NGS data have been deposited to the Sequence Read Archive in National Center for Biotechnology Information (NCBI) under the accession number PRJNA577195.

\section{Ethics Approval and Consent to Participate}

Note applicable.

\section{Consent for Publication}

The manuscript has been read and approved by all authors.

\section{Competing Interests}

The authors declare that they have no competing interests.

\section{Author details}

'Department of Biotechnology, School of Life Sciences and Technology, Center for Informational Biology, University of Electronic Science and Technology of China, Room 216, Main Building, No. 4, Section 2, North Jianshe Road, Chengdu 610054, People's Republic of China. ${ }^{2}$ Jiangsu Key Laboratory of Crop Genomics and Molecular Breeding, Agricultural College, Yangzhou University, Yangzhou 225009, China. ${ }^{3}$ Department of Plant Science and Landscape Architecture, University of Maryland, College Park, MD 20742, USA. ${ }^{4}$ Institute for Bioscience and Biotechnology Research, University of Maryland, Rockville, MD 20850, USA.
Received: 18 October 2019 Accepted: 20 January 2020

Published online: 03 February 2020

\section{References}

Akua T, Shaul O (2013) The Arabidopsis thaliana MHX gene includes an intronic element that boosts translation when localized in a 5' UTR intron. J Exp Bot 64:4255-4270

Cenik C, Derti A, Mellor JC, Berriz GF, Roth FP (2010) Genome-wide functiona analysis of human 5' untranslated region introns. Genome Biol 11:R29

Cermak T, Curtin SJ, Gil-Humanes J, Cegan R, Kono TJY et al (2017) A multipurpose toolkit to enable advanced genome engineering in plants. Plant Cell 29:1196-1217

Christian M, Qi Y, Zhang Y, Voytas DF (2013) Targeted mutagenesis of Arabidopsis thaliana using engineered TAL effector nucleases. G3 (Bethesda) 3:1697-1705

Ding D, Chen K, Chen Y, Li H, Xie K (2018) Engineering introns to express RNA guides for Cas9- and Cpf1-mediated multiplex genome editing. Mol Plant 11: $542-552$

Fonfara I, Richter H, Bratovic M, Le Rhun A, Charpentier E (2016) The CRISPRassociated DNA-cleaving enzyme Cpf1 also processes precursor CRISPR RNA. Nature 532:517-521

Gallegos JE, Rose AB (2017) Intron DNA sequences can be more important than the proximal promoter in determining the site of transcript initiation. Plant Cell 29:843-853

Gao Y, Zhao Y (2014) Self-processing of ribozyme-flanked RNAs into guide RNAs in vitro and in vivo for CRISPR-mediated genome editing. J Integr Plant Biol 56:343-349

Jeong YM, Mun JH, Lee I, Woo JC, Hong CB et al (2006) Distinct roles of the first introns on the expression of Arabidopsis profilin gene family members. Plant Physiol 140:196-209

Jiang W, Zhou H, Bi H, Fromm M, Yang B et al (2013) Demonstration of CRISPR/ Cas9/sgRNA-mediated targeted gene modification in Arabidopsis, tobacco, sorghum and rice. Nucleic Acids Res 41:e188

Jiang WZ, Weeks DP (2017) A gene-within-a-gene Cas9/sgRNA hybrid construct enables gene editing and gene replacement strategies in Chlamydomonas reinhardtii. Algal Res 26:474-480

Li JF, Norville JE, Aach J, McCormack M, Zhang D et al (2013) Multiplex and homologous recombination-mediated genome editing in Arabidopsis and Nicotiana benthamiana using guide RNA and Cas9. Nat Biotechnol 31:688-691

Lowder LG, Zhang D, Baltes NJ, Paul JW, Tang X et al (2015) A CRISPR/Cas9 toolbox for multiplexed plant genome editing and transcriptional regulation. Plant Physiol 169:971-985

Lowder LG, Zhou J, Zhang Y, Malzahn A, Zhong Z et al (2018) Robust transcriptional activation in plants using multiplexed CRISPR-Act2.0 and mTALE-act systems. Mol Plant 11:245-256

Malzahn AA, Tang X, Lee K, Ren Q, Sretenovic S et al (2019) Application of CRISPR-Cas12a temperature sensitivity for improved genome editing in rice, maize, and Arabidopsis. BMC Biol 17:9

Mikami M, Toki S, Endo M (2017) In planta processing of the SpCas9-gRNA complex. Plant Cell Physiol 58:1857-1867

Murray MG, Thompson WF (1980) Rapid isolation of high molecular weight plant DNA. Nucleic Acids Res 8:4321-4325

Nekrasov V, Staskawicz B, Weigel D, Jones JD, Kamoun S (2013) Targeted mutagenesis in the model plant Nicotiana benthamiana using Cas9 RNAguided endonuclease. Nat Biotechnol 31:691-693

Puchta H (2017) Applying CRISPR/Cas for genome engineering in plants: the best is yet to come. Curr Opin Plant Biol 36:1-8

Qi Y, Li X, Zhang Y, Starker CG, Baltes NJ et al (2013) Targeted deletion and inversion of tandemly arrayed genes in Arabidopsis thaliana using zinc finger nucleases. G3 (Bethesda) 3:1707-1715

Ren Q, Zhong Z, Wang Y, You Q, Li Q et al (2019) Bidirectional Promoter-Based CRISPR-Cas9 Systems for Plant Genome Editing. Front Plant Sci 10:1173

Shan Q, Wang Y, Chen K, Liang Z, Li J et al (2013a) Rapid and efficient gene modification in rice and Brachypodium using TALENs. Mol Plant 6:1365-1368

Shan Q, Wang Y, Li J, Zhang Y, Chen K et al (2013b) Targeted genome modification of crop plants using a CRISPR-Cas system. Nat Biotechnol 31: $686-688$

Tang X, Liu G, Zhou J, Ren Q, You Q et al (2018) A large-scale whole-genome sequencing analysis reveals highly specific genome editing by both Cas 9 and Cpf1 (Cas12a) nucleases in rice. Genome Biol 19:84 
Tang X, Lowder LG, Zhang T, Malzahn AA, Zheng X et al (2017) A CRISPR-Cpf1 system for efficient genome editing and transcriptional repression in plants. Nat Plants 3:17018

Tang X, Ren Q, Yang L, Bao Y, Zhong Z et al (2019) Single transcript unit CRISPR 2.0 systems for robust Cas9 and Cas12a mediated plant genome editing. Plant Biotechnol J 17:1431-1445

Tang X, Zheng X, Qi Y, Zhang D, Cheng Y et al (2016) A single transcript CRISPRCas9 system for efficient genome editing in plants. Mol Plant 9:1088-1091

Tsai SQ, Wyvekens N, Khayter C, Foden JA, Thapar V et al (2014) Dimeric CRISPR RNA-guided Fokl nucleases for highly specific genome editing. Nat Biotechnol 32:569-576

Voytas DF (2013) Plant genome engineering with sequence-specific nucleases. Annu Rev Plant Biol 64:327-350

Wang M, Mao Y, Lu Y, Wang Z, Tao X et al (2018) Multiplex gene editing in rice with simplified CRISPR-Cpf1 and CRISPR-Cas9 systems. J Integr Plant Biol 60: 626-631

Xie K, Minkenberg B, Yang Y (2015) Boosting CRISPR/Cas9 multiplex editing capability with the endogenous tRNA-processing system. Proc Natl Acad Sci U S A 112:3570-3575

You Q, Zhong Z, Ren Q, Hassan F, Zhang Y et al (2018) CRISPRMatch: an automatic calculation and visualization tool for high-throughput CRISPR genome-editing data analysis. Int J Biol Sci 14:858-862

Zetsche B, Heidenreich M, Mohanraju P, Fedorova I, Kneppers J et al (2017) Multiplex gene editing by CRISPR-Cpf1 using a single crRNA array. Nat Biotechnol 35:31-34

Zhang Y, Malzahn AA, Sretenovic S, Qi Y (2019) The emerging and uncultivated potential of CRISPR technology in plant science. Nat Plants 5:778-794

Zhang Y, Zhang F, Li X, Baller JA, Qi Y et al (2013) Transcription activator-like effector nucleases enable efficient plant genome engineering. Plant Physiol 161:20-27

Zheng X, Yang S, Zhang D, Zhong Z, Tang X et al (2016) Effective screen of CRISPR/Cas9-induced mutants in rice by single-strand conformation polymorphism. Plant Cell Rep 35:1545-1554

Zhong Z, Sretenovic S, Ren Q, Yang L, Bao Y et al (2019) Improving plant genome editing with high-Fidelity $\times$ Cas9 and non-canonical PAM-targeting Cas9-NG. Mol Plant 12:1027-1036

Zhong Z, Zhang Y, You Q, Tang X, Ren Q et al (2018) Plant genome editing using FnCpf1 and LbCpf1 nucleases at redefined and altered pam sites. Mol Plant 11:999-1002

Zhou J, Deng K, Cheng Y, Zhong Z, Tian L et al (2017) CRISPR-Cas9 based genome editing reveals new insights into MicroRNA function and regulation in rice. Front Plant Sci 8:1598

Zhou J, Xin X, He Y, Chen H, Li Q et al (2019) Multiplex QTL editing of grainrelated genes improves yield in elite rice varieties. Plant Cell Rep 38:475-485

\section{Publisher's Note}

Springer Nature remains neutral with regard to jurisdictional claims in published maps and institutional affiliations.

\section{Submit your manuscript to a SpringerOpen ${ }^{\circ}$ journal and benefit from:}

- Convenient online submission

- Rigorous peer review

- Open access: articles freely available online

- High visibility within the field

- Retaining the copyright to your article

Submit your next manuscript at $\boldsymbol{\nabla}$ springeropen.com 